\title{
A Machian Request for the Equivalence Principle in Extended Gravity and non-geodesic motion
}

\author{
${ }^{1}$ Ignazio Licata, ${ }^{2}$ Christian Corda, ${ }^{3}$ Elmo Benedetto
}

May 11, 2018

\begin{abstract}
${ }^{1}$ Institute for Scientific Methodology, Palermo, Italy; School of Advanced International Studies on Theoretical and non Linear Methodologies of Physics, Bari, I-70124, Italy; e-mail: ignaziolicata@ejtp.info;

${ }^{2}$ Dipartimento di Fisica, Scuola Superiore di Studi, Universitari e Ricerca 'Santa Rita', Via Tagliamento 45, 00188 Roma, Italy; Austro-Ukrainian Institute for Science and Technology, Institut für Theoretishe Physik, Technische Universität, Wiedner Hauptstrasse 8-10/136, A-1040, Wien, Austria; International Institute for Applicable Mathematics \& Information Sciences (IIAMIS), B.M. Birla Science Centre, Adarsh Nagar, Hyderabad- 500 463, India; e-mail: cordac.galilei@gmail.com;

${ }^{3}$ Department of Engineering, University of Sannio, Piazza Roma 21, 82100 Benevento, Italy; e-mail: elmobenedetto@libero.it; orcid: 0000-0002-5920-0221;
\end{abstract}

\begin{abstract}
Starting from the origin of Einstein general relativity (GR) the request of Mach on the theory's structure has been the core of the foundational debate. That problem is strictly connected with the nature of the mass-energy equivalence. It is well known that this is exactly the key point that Einstein used to realize a metric theory of gravitation having an unequalled beauty and elegance. On the other hand, the current requirements of particle physics and the open questions within extended gravity theories request a better understanding of Equivalence Principle (EP). The MOND theory by Milgrom proposes a modification of Newtonian dynamics and we consider a direct coupling between the Ricci curvature scalar and the matter Lagrangian showing that a non geodesic ratio $m_{i} / m_{g}$ can be fixed and that Milgrom acceleration is retrieved at low energies.
\end{abstract}

\section{Introduction}

It is well known that The Science of Mechanics by Ernst Mach had a strong influence on Einstein and played an important role in the development of GR 
[1. In Newton's Philosophiae Naturalis Principia Mathematica, accelleration is considered as absolute. In the famous Gedankenexperiment of the rotating bucket filled with water, Newton deduced the existence of an absolute rotation by observing the curved surfaces on the water.

The aim of Newton was to explain the inertia through a sort of resistance to motion in the absolute space which, in this way, comes to be an agent and not a mere physical theater of coordinates, although unspecified. The first thinker to question the Newtonian reasoning was the philosopher George Berkeley in his De Motu, published in 1721 and he can be considered the precursor of Mach. Indeed, after more than 150 years, Mach proposed a radical criticism of Newton's absolute space and he concluded that the inertia would be an interaction that requires other bodies to manifest itself, so that it would make no sense in a Universe consisting of just a single mass. According to Mach, there is a total relational symmetry and every motion, uniform or accelerated, makes sense only in reference to other bodies. Therefore, following the so called Mach Principle, the inertia of a body is not an intrinsic property and depends on the mass distribution in the rest of the Universe. Einstein was very fascinated by Mach reasoning but it is widely acknowledged that Mach Principle is not fully incorporated into relativistic field equations [2]. The challenge of a Machian physics was accepted several times (though less than expected) in the context of both classical and quantum. Here we recall the Narlikar's theory with variable mass derived from Wheeler-Feynman-like action at a distance theory [3, 4. Sciama's theory requires to get the inertia as "gravitational closeness" (and the perfect equivalence) under the precise cosmological condition $G \rho \frac{r^{2}}{c^{2}}=1$ where $r$ is the radius of the universe, $\rho$ the density, $c$ is the speed of light and $G$ the gravi-

tational constant [5. In quantum contexts, and in Higgs times, the problem becomes more complex [6, 7, 8, 9, 10].

\section{The Physics Under the Metric}

Einstein has often stated that some Machian effects are present in GR. In particular, in the famous Lectures of 1921 [11] he states that it showed the following effects:

1) The inertia of a body must increase when ponderable masses are piled up in its neighbourhood.

2) A body must experience an accelerating force when neighboring masses are accelerated and the force must be in the same direction as that acceleration.

3) A rotating hollow body must generate inside of itself a Coriolis field which deflects moving bodies in the sense of the rotation and a radial centrifugal field as well.

Let us follow the Einstein reasoning. By considering the geodesic equation

$$
\frac{d^{2} x_{\mu}}{d s^{2}}+\Gamma_{\alpha \beta}^{\mu} \frac{d x_{\alpha}}{d s} \frac{d x_{\beta}}{d s}=0
$$


Einstein worked in the weak- field approximation and the metric he found to represent the gravitational field due to a distribution of small masses corresponding to a density $\sigma$ and having small velocities $\frac{d x_{i}}{d s}$ can be written as

$$
\begin{gathered}
g_{00}=1-\frac{2 G}{c^{2}} \int \frac{\sigma d V}{r} \\
g_{0 i}=\frac{4 G}{c^{2}} \int \frac{d x_{i}}{d s} \frac{\sigma d V}{r} \\
g_{i j}=-\delta_{i j}\left(1+\frac{2 G}{c^{2}} \int \frac{\sigma d V}{r}\right)
\end{gathered}
$$

The equation of motion in this field becomes

$$
\frac{d}{d x^{0}}[(1+\bar{\sigma}) v]=\nabla \bar{\sigma}+\frac{\partial A}{\partial x^{0}}+(\nabla \wedge A) \wedge v
$$

where

$$
\begin{gathered}
\bar{\sigma} \equiv \frac{G}{c^{2}} \int \frac{\sigma d V}{r} \\
A=\frac{4 G}{c^{2}} \int \frac{\sigma v d V}{r} .
\end{gathered}
$$

Einstein interpreted it by saying that the inertial mass is proportional to $1+\bar{\sigma}$ and therefore increases when ponderable masses approach the test body

$$
m_{i}=m_{g}\left(1+\frac{G}{c^{2}} \int \frac{\bar{\sigma} d V}{r}\right)
$$

Many physicists believe, according to C. Brans [12, that only the second and third effect are contained in GR. At first glance it seems that, if the Einstein interpretation is right, the EP is violated but we emphasize that all bodies with different inertial masses are still falling with the same acceleration in a gravitational field. In [13] the author analyzes what he calls Modified Mach Principle in the context of an expanding universe. He suggests the following definitions for the inertial mass within and beyond the bulge of galaxies as

$$
\begin{array}{ccc}
m_{i}=C & r \leq R_{0} \\
m_{i}=\frac{C^{\prime}}{r}=m_{g} \frac{R_{0}}{r} & r>R_{0}
\end{array}
$$

where $C$ and $C^{\prime}$ are constants and he calls the first one as inertial mass versus gravitational interaction within the bulge, and the second one as inertial mass versus cosmological expansion beyond the bulge. It would seem that the introduction of a genuine Mach's principle implies a re-introduction of the distinction between inertial mass and gravitational mass, hidden under the metric of GR and the strong form of the EP. Let recall that the equivalence between $m_{i}$ and $m_{g}$ is the axiomatic and constructive keystone of GR. This raises the problem of the interpretation of the formalism able to establish the EP on the physical meaning of the relationship between $m_{i}$ and $m_{g}$. 


\section{Inertial and Gravitational Mass}

The nature of dark matter is one of the unsolved mysteries in cosmology since C. Zwicky measured the velocity dispersion of the Coma cluster of galaxies [14]. Let us rewrite the following relation

$$
m_{i} \frac{v^{2}}{r}=\frac{G M_{g} m_{g}}{r^{2}}
$$

where $m_{i}$ is a body that rotates around a gravitational mass $M_{g}$ over a constant radius $r$. The relation

$$
v=\sqrt[4]{G M a_{0}}
$$

is in perfect agreement with the experimental data with $a_{0}$ is about $10^{-10} \frac{\mathrm{m}}{\mathrm{s}^{2}}$ [15] - 23. Therefore we write

$$
v^{2}=\frac{G M_{g}}{r} \frac{m_{g}}{m_{i}}=\sqrt{G M_{g} a_{0}}
$$

It follows that

$$
\frac{m_{g}}{m_{i}}=\sqrt{\frac{a_{0} r^{2}}{G M_{g}}}
$$

If we do not interpret $a_{0}$ from the kinematic point of view but as a gravitational field, we can write

$$
\frac{m_{g}}{m_{i}}=\sqrt{\frac{g_{0}}{g}} .
$$

According to Mach and his interpreters, the inertial mass of a body arises as a consequence of its interactions with the Universe and so we assume that

$$
\frac{m_{g}}{m_{i}}=\mu(x)
$$

with $\mu=1$ for $\left|\frac{g}{g_{0}}\right| \gg 1$ and $\mu=\sqrt{ }$ for $\left|\frac{g}{g_{0}}\right| \ll 1$.

A possible form of $\mu$ may be for example

$$
\mu=\sqrt{\frac{g_{0}+g}{g}}
$$

where $g$ is the field generated by nearby masses.

It is easy to verify that when $g \gg g_{0}$, circular velocity decreases in Keplerian way but when $g \ll g_{0}$ we obtain

$$
v^{2}=\frac{G M_{g}}{r} \sqrt{\frac{g_{0}}{g}}=G M_{g} \sqrt{\frac{g_{0}}{G M_{g}}}=\sqrt{G M_{g} g_{0}}
$$

and finally 


$$
v=\sqrt[4]{G M g_{0}}
$$

Obviously the value of $g_{0}$ that fits all the data of galaxies rotation curves is about $10^{-10} \mathrm{~m} / \mathrm{s}^{2}$. From the mathematical point of view the relations (8) and (15) coincide but from a physical point of view the situation is different. At every point in the Universe, the second Newtonian law is still valid even for small accelerations.

Deviations between inertial and gravitational mass as stressed by eq. (10) can have an intriguing geometrical explanation in the framework of $f(R)$ theories of gravity, assuming an explicit coupling between an arbitrary function of the scalar curvature, $R$, and the Lagrangian density of matter [25. The most simple situation is to consider a weak modification of general relativity, which could be consistent with solar system tests, which implies a direct coupling between the Ricci curvature scalar and the matter Lagrangian [26]. Following [25, 26], let us consider the action (for the sake of simplicity we set $16 \pi G=1, c=1$ and $\hbar=1$ hereafter)

$$
S=\int d^{4} x \sqrt{-g}\left(R+\lambda R \mathcal{L}_{m}+\mathcal{L}_{m}\right),
$$

which only includes a coupling between the Ricci scalar and the matter Lagrangian, being $\lambda$ the coupling constant, with respect to the well known canonical Einstein - Hilbert action of standard general relativity [27]

$$
S=\int d^{4} x \sqrt{-g}
$$

Without loss of generality, we can also set $\lambda=1$. Thus, the standard variation analysis in a local Lorentz frame enables to write [25, 26]

$$
\begin{aligned}
& \delta \int d^{4} x \sqrt{-g}\left(R+R \mathcal{L}_{m}+\mathcal{L}_{m}\right)=\int d^{4} x\left[\delta \sqrt{-g}\left(R+R \mathcal{L}_{m}+\mathcal{L}_{m}\right)+\sqrt{-g} \delta\left(R+R \mathcal{L}_{m}+\mathcal{L}_{m}\right)\right] \\
& \quad=\int d^{4} x\left[\sqrt{-g}\left(1+\mathcal{L}_{m}\right) R_{\mu \nu}-\frac{1}{2} g_{\mu \nu}\left(R+R \mathcal{L}_{m}+\mathcal{L}_{m}\right)\right] g^{\mu \nu}+d^{4} x \sqrt{-g}\left(1+\mathcal{L}_{m}\right) g^{\mu \nu} \delta R_{\mu \nu}
\end{aligned}
$$

The relation between the connections and the Ricci tensor [27] gives [25, 26]

$$
g^{\mu \nu} \delta R_{\mu \nu}=g^{\mu \nu} \partial_{\alpha}\left(\delta \Gamma_{\mu \nu}^{\alpha}\right)-g^{\mu \alpha} \partial_{\alpha}\left(\delta \Gamma_{\mu \nu}^{\nu}\right) \equiv \partial_{\alpha} X^{\alpha},
$$

where

$$
X^{\alpha} \equiv g^{\mu \nu}\left(\delta \Gamma_{\mu \nu}^{\alpha}\right)-g^{\mu \alpha}\left(\delta \Gamma_{\mu \nu}^{\nu}\right) .
$$

Thus, the second integral in equation (18) results to be [25, 26]

$$
\begin{aligned}
& \int d^{4} x \sqrt{-g}\left(1+\mathcal{L}_{m}\right) g^{\mu \nu} \delta R_{\mu \nu}=\int d^{4} x \sqrt{-g}\left(1+\mathcal{L}_{m}\right) \partial_{\alpha} X^{\alpha}= \\
& =\int d^{4} x \partial_{\alpha}\left[\sqrt{-g}\left(1+\mathcal{L}_{m}\right) X^{\alpha}\right]-\int d^{4} x \partial_{\alpha}\left[\sqrt{-g}\left(1+\mathcal{L}_{m}\right)\right] X^{\alpha}
\end{aligned}
$$

A standard assumption is that fields are equal to zero at infinity. Then we obtain [25, 26] 


$$
d^{4} x \sqrt{-g}\left(1+\mathcal{L}_{m}\right) g^{\mu \nu} \delta R_{\mu \nu}=-\int d^{4} x \partial_{\alpha}\left[\sqrt{-g}\left(1+\mathcal{L}_{m}\right)\right] X^{\alpha} .
$$

Let us calculate the quantity $X^{\alpha}$. In a local Lorentz frame it is [25, 26]

$$
\nabla \beta g_{\mu \nu}=\partial_{\beta} g_{\mu \nu}=0 .
$$

Thus, the well known definitions of the Christofell connections [27] gives [25, 26]

$$
\begin{gathered}
\delta \Gamma_{\mu \nu}^{\alpha}=\delta\left[\frac { 1 } { 2 } g ^ { \beta \alpha } \left(\partial_{\mu}\right.\right. \\
=\frac{1}{2} g^{\beta \alpha}\left(\partial_{\mu} \delta_{\beta \nu}+\partial_{\nu} \delta_{\mu \beta}-\partial_{\beta} \delta_{\mu \nu}\right) .
\end{gathered}
$$

In analogous way one gets 25,26 ,

$$
\delta \Gamma_{\mu \nu}^{\nu}=\frac{1}{2} g^{\nu \beta} \partial_{\mu}\left(\delta g_{\nu \beta}\right) .
$$

Using eqs. (24) and (25) we find [25, 26]

$$
g^{\mu \nu}\left(\delta \Gamma_{\mu \nu}^{\alpha}\right)=\frac{1}{2} \partial^{\alpha}\left(g_{\mu \nu} \delta g^{\mu \nu}\right)-\partial^{\mu}\left(g_{\beta \mu} \delta g^{\nu \beta}\right)
$$

and

$$
g^{\mu \alpha}\left(\delta \Gamma_{\mu \nu}^{\nu}\right)=-\frac{1}{2} \partial^{\alpha}\left(g \delta g^{\nu \beta}\right)
$$

Now, substituting in (20), we obtain [25, 26 ,

$$
X^{\alpha}=\partial^{\alpha}\left(g_{\mu \nu} \delta g^{\mu \nu}\right)-\partial^{\mu}\left(g_{\mu \nu} \delta g^{\alpha \nu}\right) .
$$

In this way, equation (22) becomes [25, 26]

$$
\begin{gathered}
\int d^{4} x \sqrt{-g}\left(1+\mathcal{L}_{m}\right) g^{\mu \nu} \delta R_{\mu \nu}= \\
=\int d^{4} x \partial_{\alpha}\left[\sqrt{-g}\left(1+\mathcal{L}_{m}\right)\right]\left[\partial\left(g_{\mu \nu} \delta g^{\alpha \nu}\right)-\partial^{\alpha}\left(g \delta g^{\nu \beta}\right],\right.
\end{gathered}
$$

which also gives [25, 26]

$$
\begin{gathered}
\int d^{4} x \sqrt{-g}\left(1+\mathcal{L}_{m}\right) g^{\mu \nu} \delta R_{\mu \nu}= \\
=\int d^{4} x\left\{g _ { \mu \nu } \partial ^ { \alpha } \partial _ { \alpha } \left[\sqrt{-g}(1+\}-\int d^{4} x\left\{g _ { \mu \nu } \partial ^ { \mu } \partial _ { \alpha } \left[\sqrt{-g}\left(1+\mathcal{L}_{m} \delta g^{\alpha \nu}\right\} .\right.\right.\right.\right.
\end{gathered}
$$

Inserting eq. (30) in the variation (18) we get

$$
\begin{gathered}
\delta \int d^{4} x \sqrt{-g}\left(R+R \mathcal{L}_{m}+\mathcal{L}_{m}\right)=\int d^{4} x\left[\sqrt{-g}\left(1+\mathcal{L}_{m}\right) R_{\mu \nu}-\frac{1}{2} g_{\mu \nu}\left(R+R \mathcal{L}_{m}+\mathcal{L}_{m}\right)\right] \delta g^{\mu \nu}+ \\
\int d^{4} x\left\{g_{\mu \nu} \partial^{\alpha} \partial_{\alpha}\left[\sqrt{-g}\left(1+\mathcal{L}_{m}\right)\right]-g_{\alpha \nu} \partial^{\mu} \partial_{\alpha}\left[\sqrt{-g}\left(1+\mathcal{L}_{m}\right)\right] \delta g_{\mu \nu}\right\}+d^{4} x\left\{(1+R) \delta\left(\sqrt{-g} \mathcal{L}_{m}\right)\right\} .
\end{gathered}
$$

This variation is equal to zero for [25, 26] 


$$
R_{\mu \nu}-\frac{1}{2} R g_{\mu \nu}=-\mathcal{L}_{m} R_{\mu \nu}+\left(\nabla_{\mu} \nabla_{\nu}-g_{\mu \nu} \square\right) \mathcal{L}_{m}+\frac{(1+R)}{2} T_{\mu \nu}^{(m)}
$$

which are the Einstein field equations modified by direct coupling between the Ricci curvature scalar and the matter Lagrangian. In fact, the standard stressenergy tensor [27]

$$
T_{\mu \nu}^{(m)} \equiv \frac{-2}{\sqrt{-g}} \frac{\delta(\sqrt{-g} \mathcal{L}}{\delta g^{\mu \nu}}
$$

has been introduced in the modified field equations (32). Writing down explicitly the Einstein tensor and introducing a "total" stress-energy tensor [25, 26, 28]

$$
T_{\mu \nu}^{(t o t)} \equiv \frac{1}{\left(1+\mathcal{L}_{m}\right)}\left[\left(\nabla \mu \nabla \nu-g_{\mu \nu} \square\right) \mathcal{L}_{m}+\frac{(1+R)}{2} T_{\mu \nu}^{(m)}-\frac{R \mathcal{L}_{m}}{2} g_{\mu \nu}\right]
$$

eqs. (32) can be put in the well known Einsteinian form

$$
G_{\mu \nu}=\frac{1}{2} T_{\mu \nu}^{(t o t)},
$$

in which a curvature contribution 28 is added and mixed to the material one. In other words, the high order terms contribute, like sources, to the modified field equations and have to be considered like effective fields ([28] for details). The condition of energy conservation [25, 26, 27]

$$
\nabla^{\mu} G_{\mu \nu}=0
$$

can be inserted in eqs. (35) and (34), obtaining

$$
\nabla^{\mu} T_{\mu \nu}^{(m)}=\frac{1}{R+1}\left(g_{\mu \nu}\right.
$$

Now, we can introduce the well known stress-energy tensor of a perfect fluid [27.

$$
T_{\mu \nu}^{(m)} \equiv(\epsilon+p) u_{\mu} u_{\nu}-p g_{\mu \nu}
$$

in order to test the motion of test particles [25, 26], where $\epsilon$ is the proper energy density, $p$ the pressure and $u_{\mu}$ the fourth-velocity of the particles. This is the simplest version of a stress-energy tensor for the matter, concerning inchoerent matter, and it is considered a good approximation in astrophysics frameworks [25, 26, 27]. Introducing the projector operator [25, 26]

$$
P_{\mu \alpha} \equiv g_{\mu \alpha}-u_{\mu} u_{\alpha}
$$

we can apply the contraction $g^{\alpha \beta} P_{\mu \beta}$ to equation (37), obtaining [25, 26]

$$
\frac{d^{2} x_{\mu}}{d s^{2}}+\Gamma_{\alpha \beta}^{\mu} \frac{d x_{\alpha}}{d s} \frac{d x_{\beta}}{d s}=F^{\alpha}
$$


Thus, we find the existence of an extra force [25, 26]

$$
F^{\alpha} \equiv(\epsilon+p)^{-1} P^{\alpha \nu}\left[\left(\frac{1}{R+1}\right)\left(\mathcal{L}_{m}+p\right) \nabla_{\mu} R+\nabla_{\mu} p\right]
$$

showing that the motion of test particles is $\backslash$ emph\{non-geodesic\}. This extra force is orthogonal to the four-velocity of test masses [25, 26]

$$
F^{\alpha} \frac{d x_{\alpha}}{d s}=0 .
$$

The Newtonian limit in three dimensions of equation (40) reads [25, 26]

$$
\vec{a}_{t o t}=\vec{a}_{n}+\vec{a}_{n g} .
$$

The total acceleration $\vec{a}_{t o t}$ is given by the ordinary Newtonian acceleration $\vec{a}_{n}$ plus the repulsive acceleration $\vec{a}_{n g}$ due to the extra (non-geodesic) force [25, 26]. Eq. (43) and a bit of three-dimensional geometry permit to write the Newtonian acceleration $\vec{a}_{n}$ as $[25,26]$

$$
\vec{a}_{n}=\frac{1}{2}\left(a_{t o t}^{2}-a_{n}^{2}-a_{n g}^{2}\right) \frac{\vec{a}_{t o t}}{a_{n g} a_{t o t}}
$$

Considering the limit in which $\vec{a}_{n g}$ dominates (i.e. $a_{n} \ll a_{t o t}$ ) one gets

$$
a_{n} \simeq \frac{a_{t o t} \vec{a}_{t o t}}{2 a_{n g}}\left(1-\frac{a_{n g}^{2}}{a_{t o t}^{2}}\right) .
$$

Thus, the extra aceleration is given by [25, 26]

$$
a_{0} \equiv\left[\frac{1}{2 a_{n g}}\left(1-\frac{a_{n g}^{2}}{a_{t o t}^{2}}\right)\right]^{-1},
$$

and combining eq. (46) with eqs. (10) and (11) one gets

$$
\frac{m_{g}}{m_{i}}=\sqrt{\frac{g_{0}}{g}}=\sqrt{\frac{r^{2}}{\frac{M_{g}}{2 a_{n g}}\left(1-\frac{a_{n g}^{2}}{a_{t o t}^{2}}\right)}}
$$

with

$$
g_{0}=\frac{r^{2}}{\frac{1}{2 a_{n g}}\left(1-\frac{a_{n g}^{2}}{a_{t o t}^{2}}\right)} .
$$

Thus, we have shown that in our model the ratio between gravitational and inertial mass is explained in an elegant, geometric way through a direct coupling between the Ricci curvature scalar and the matter Lagrangian which generates a non geodesic motion of test particles. 


\section{Conclusions}

The assumption of an $R$-dependent inertial mass is in accordance with the spirit of Mach principle and Einstein himself tried to implement this hypothesis in the context of General Relativity [29]. The possible relation $m_{g} / m_{i}$ is deduced by comparing it with Milgrom's rotational equation that is in perfect agreement with the experimental data. However, the interpretation given here is different, and it leaves unchanged in any point the second law of dynamics. Finally we have shown that the ratio between gravitational and inertial mass is explained in geometric way through a direct coupling between the Ricci curvature scalar and the matter Lagrangian which generates a non geodesic motion of test particles.

Such a non geodesic motion is due to the presence of the centrifugal extra force in eq. (42).

\section{Acknowledgements}

The authors thank an unknown referees for useful comments.

\section{References}

[1] Mach E., 1883, Die Mechanik in ihrer Entwicklung Historisch-Kritisch Dargerstellt, Leipzig: Brockhaus.

[2] H. Ohanian, R. Ruffini "Gravitation and Spacetime", W.W. Norton, New York (1994).

[3] J. V. Narlikar, Action at a distance and cosmology: A historical perspective, Annual Review of Astronomy and Astrophysics, 41, (2003): 169-189.

[4] J. V. Narlikar, Inertia and cosmology in Einstein's relativity, in Relativity, Quanta and Cosmology, Einstein Centenary Vol. II. Eds. M. Pantaleo and F. de Finis, Johnson Reprint Corporation, 493 (1979).

[5] D. Sciama, On the Origin of Inertia, Monthly Notices of the Royal Astronomical Society, Vol. 113 (1953):34- 42.

[6] J. Barbour, H. Pfister, Mach's Principle: From Newton's Bucket to Quantum Gravity, Birkhauser, 1995.

[7] H. C. Rosu, Classical and Quantum Inertia: A Matter of Principle, Gravitation and Cosmology vol. 5 No. 2 (1999) 81-91.

[8] A. B. Arbuzov, L. A. Glinka, V. N. Pervushin, Higgs Particle Mass in Cosmology, arXiv:0705.4672 [hep-ph], 2008.

[9] Y. N. Srivastava, J. Swain, A. Widom, An Argument for Nonminimal Higgs Coupling to Gravity, arXiv:1110.5549 [gr-qc], 2011. 
[10] Y. N. Srivastava, A. Widom, Gravitational Decay Modes of the Standard Model Higgs Particle, arXiv:hep-ph/0003311, 2000.

[11] Einstein, A., The Meaning of Relativity. Four lectures delivered at Princeton University, May, 1921, Princeton Univ. Press, 2004.

[12] Brans C.H. "Mach's Principle and the Locally Measured Gravitational Constant in General Relativity" Phys. Rev. Vol. 125, 388 (1962).

[13] F. Darabi "Is flat rotation curve a sign of cosmic expansion?" MNRAS 433, (2013).

[14] F. Zwicky, Spectral displacement of extra galactic nebulae, Helv. Phys. Acta. 6, 110-127 (1933).

[15] M. Milgrom "A modification of the Newtonian dynamics as a possible alternative to the hidden mass hypothesis". Astrophysical Journal 270: 365-370 (1983).

[16] M. Milgrom "A modification of the Newtonian dynamics - Implications for galaxies" Astrophysical Journal 270: 371-389 (1983).

[17] M. Milgrom, "MOND theory", Canadian Journal of Physics, e-First Article: pp. 1-12, doi: 10.1139/cjp-2014-0211 (2014).

[18] M. Milgrom "MD or DM? Modified dynamics at low accelerations vs dark matter". Proceedings of Science (2011).

[19] V.A. De Lorenci, M. Faundez-Abans, J.P. Pereira "Testing the Newton second law in the regime of small accelerations" Astronomy \& Astrophysics, Vol. 503, N. 1 (2009).

[20] K.G. Begeman, A.H. Broeils, R.H. Sanders "Extended rotation curves of spiral galaxies: dark haloes and modified dynamics" MNRAS 249, 523 (1991).

[21] J. Bekenstein, M. Milgrom "Does the missing mass problem signal the breakdown of Newtonian gravity?" Astrophysical Journal 286, 7-14 (1984).

[22] W. J. G de Blok, S. S. McGaugh "Testing Modified Newtonian Dynamics with Low Surface Brightness Galaxies: Rotation Curve Fits" Astrophysical Journal 508, 132-140 (1998).

[23] Mortlock, D. J., and Turner, E. L."Gravitational lensing in modified Newtonian dynamics" MNRAS 327, 557 (2001).

[24] Das S., Patitsas S. N. "Can MOND type hypotheses be tested in a free fall laboratory environment?" Physical Review D. 87, 107101 (2013).

[25] O. Bertolami, C. G. Bohmer, T. Harko and F. S. M. Lobo, Phys. Rev. D 75,104016 (2007). 
[26] C. Corda, Int. J. Theor. Phys. 47, 2679 ((2008).

[27] L. Landau L and E. Lifsits, Classical Theory of Fields (3rd ed.). London: Pergamon. ISBN 0-08-016019-0. Vol. 2 of the Course of Theoretical Physics (1971).

[28] C. Corda, N. Adv. Phys. 7, 1, 67 (2013).

[29] A. Pais, Subtle Is the Lord: The Science and the Life of Albert Einstein", Oxford University Press (2005). 